\title{
Longitudinal Assessment of Antipneumococcal Susceptibility in the United States
}

\author{
Mark E. Jones, ${ }^{1 *}$ James A. Karlowsky, ${ }^{2}$ Renée Blosser-Middleton, ${ }^{2}$ Ian A. Critchley, ${ }^{2}$ \\ Elena Karginova, ${ }^{2}$ Clyde Thornsberry, ${ }^{3}$ and Daniel F. Sahm ${ }^{2}$ \\ Focus Technologies, Inc., 1217 KP Hilversum, The Netherlands'; Focus Technologies, Inc., Herndon, \\ Virginia 201712; and Focus Technologies, Inc., Franklin, Tennessee, $37064^{3}$
}

Received 27 June 2001/Returned for modification 15 October 2001/Accepted 2 May 2002

\begin{abstract}
The prevalence of antimicrobial resistance among 4,940 U.S. pneumococcal isolates collected during 1999 was as follows: penicillin, $16.2 \%$; amoxicillin-clavulanate, $12.2 \%$; cefuroxime, $28.1 \%$; ceftriaxone, $3.6 \%$; trimethoprim-sulfamethoxazole, $30.3 \%$; azithromycin, $21.4 \%$; levofloxacin, $0.6 \%$; and moxifloxacin, $0.1 \%$. Compared to the previous 1997-1998 study (Jones et al., Antimicrob. Agents Chemother. 44:2645-2652, 2000), increases were noted for resistance to penicillin $(3.7 \% ; P<0.001)$, amoxicillin-clavulanate $(3.9 \% ; P<0.001)$, cefuroxime $(5.7 \% ; P<0.001)$, azithromycin $(2.4 \% ; P=0.014)$, trimethoprim-sulfamethoxazole $(15.4 \% ; P<$ $0.001)$, and levofloxacin $(0.3 \% ; P=0.017)$. Resistance to ceftriaxone $(0.1 \% ; P=0.809)$ and moxifloxacin $(0.03 \% ; P=0.570)$ decreased. Concurrently, multidrug resistance increased $(P<0.001)$ from $6.3 \%$ to $11.3 \%$.
\end{abstract}

Community-acquired respiratory tract infections (RTIs), from which Streptococcus pneumoniae is the most frequently isolated bacterial pathogen, are a major cause of disease in the United States (1). Antibiotic resistance in this species is a significant issue $(5,6,12,25,26)$. Except in some regions (9), resistance to more recently introduced fluoroquinolones used to treat RTIs, such as moxifloxacin, gatifloxacin, and levofloxacin, is rare $(6,12,19,25,26)$. Some reports suggest resistance to ciprofloxacin $(2,8)$ is increasing, although this is not always clear (21). We report data on the in vitro activity of moxifloxacin, levofloxacin, and other antimicrobials potentially used against $S$. pneumoniae from the second phase (1999) of a multiyear surveillance program started in 1997-1998 (12).

During 1999, non-repeat isolates of $S$. pneumoniae were collected from 290 hospital laboratories throughout the nine U.S. Bureau of the Census regions. There were 156 of 290 $(53.8 \%)$ sites that had also participated in the 1997-1998 study and had submitted at least $10 \mathrm{~S}$. pneumoniae in each study (12). Protocols for organism collection and reidentification were identical to those described previously (12). Except for the substitution of azithromycin for erythromycin and the addition of vancomycin, the antimicrobials tested remained the same and were susceptibility tested by broth microdilution according to the recommendations of the National Committee for Clinical Laboratory Standards (NCCLS) $(15,17)$. A subselection of strains included in this study were also retested as part of a faropenem comparative study (3).

The 156 hospital sites participating in both studies were included in the longitudinal data analysis. Statistical analyses were performed with the chi-square test or Wilcoxon's rank sum test (testing the null hypothesis that the median MIC distributions between years for each drug are the same). $P$ values $<0.05$ were considered statistically significant. Nucleotide sequences of quinolone-resistance-determining regions

\footnotetext{
* Corresponding author. Mailing address: Focus Technologies, Koninginneweg 11, $1217 \mathrm{KP}$ Hilversum, The Netherlands. Phone: 3135 625 7290. Fax: 3135625 7287. E-mail: mjones@focusanswers.com.
}

(QRDRs) of $g y r A, \operatorname{gyr} B$, parC, and parE and pulsed-field gel electrophoresis (PFGE) $(11,13)$ were undertaken for isolates for which levofloxacin MICs were $\geq 4 \mu \mathrm{g} / \mathrm{ml}$ or moxifloxacin MICs were $\geq 2 \mu \mathrm{g} / \mathrm{ml}$. PFGE results were analyzed with Molecular Analyst software (Bio-Rad, Hercules, Calif.) with gels normalized using S. pneumoniae R6 (ATCC 27336) restricted with SmaI (standard gel image supplied by L. McDougal, Centers for Disease Control and Prevention, Atlanta, Ga.). Percentages of similarity between isolates were calculated with the Dice coefficient.

The overall susceptibility profiles for $S$. pneumoniae isolates categorized according to penicillin susceptibility from all 290 sites are given (Table 1). Table 2 shows a longitudinal comparison of susceptibility data from the 156 sites that participated in both the 1997-1998 and 1999 studies. Overall increases in nonsusceptibility were recorded for all agents: amoxicillin-clavulanate, $5.3 \%$; cefuroxime, $4.2 \%$; ceftriaxone, $3.4 \%$; azithromycin, 3.4\%; trimethoprim-sulfamethoxazole (SXT), 6.7\%; penicillin, 1.0\%; levofloxacin, 0.4\%; and moxifloxacin, $0.3 \%$. Significant increases in resistant isolates were recorded for penicillin $(P<0.001)$, amoxicillin-clavulanate $(P$ $<0.001)$, cefuroxime $(P<0.001)$, SXT $(P<0.001)$, azithromycin $(P=0.014)$, and levofloxacin $(P=0.017)$. For levofloxacin, this represented an increase to 31 resistant strains from 9 strains in 1997-1998. Two isolates showed high-level penicillin resistance (MICs of $>32$ and $16 \mu \mathrm{g} / \mathrm{ml}$, respectively) recovered from hospitals in the West North Central and Pacific regions.

Similar to the 1997-1998 study (12), fluoroquinolone resistance remained independent of resistance to other agents (including penicillin and macrolides) (Table 1). Of the 31 isolates resistant to levofloxacin, 6 remained susceptible to moxifloxacin (20 intermediate, 5 resistant). Comparative fluoroquinolone MIC distributions for both studies are shown (Fig. 1). Statistical tests show that the differences in MIC distributions per drug per year appear significant for both agents $(P<$ $0.001)$, although for levofloxacin, this represents a shift towards a raised median MIC, and for moxifloxacin, it represents a shift towards a lower median MIC. While the number of levofloxa- 
TABLE 1. Susceptibility of all S. pneumoniae isolates to antimicrobials according to penicillin susceptibility status $(n=4,940)$ from 1999

\begin{tabular}{|c|c|c|c|c|c|c|c|}
\hline \multirow{2}{*}{$\begin{array}{l}\text { Antimicrobial agent and penicillin } \\
\text { susceptibility status }{ }^{a}\end{array}$} & \multicolumn{4}{|c|}{$\operatorname{MIC}(\mu \mathrm{g} / \mathrm{ml})^{g}$} & \multicolumn{3}{|c|}{$\%$ of isolates ${ }^{b}:$} \\
\hline & Range & $50 \%$ & $90 \%$ & Mode & $\mathrm{S}$ & I & $\mathrm{R}$ \\
\hline \multicolumn{8}{|l|}{ Penicillin } \\
\hline All & $\leq 0.06->32$ & $\leq 0.06$ & 2 & $\leq 0.06$ & 64.6 & 19.3 & 16.2 \\
\hline Pen-S & $\leq 0.06$ & $\leq 0.06$ & $\leq 0.06$ & $\leq 0.06$ & 100 & 0 & 0 \\
\hline Pen-I & $0.12-1$ & 0.5 & 1 & 1 & 0 & 100 & 0 \\
\hline Pen-R & $2->32$ & 2 & 4 & 2 & 0 & 0 & 100 \\
\hline \multicolumn{8}{|l|}{ Amoxicillin-clavulanate ${ }^{c}$} \\
\hline All & $\leq 0.03-16$ & $\leq 0.03$ & 2 & $\leq 0.03$ & $77.9(94.6)$ & $9.9(3.4)$ & $12.2(2.0)$ \\
\hline Pen-S & $\leq 0.03-0.25$ & $\leq 0.03$ & $\leq 0.03$ & $\leq 0.03$ & $100(100)$ & $0(0)$ & $0(0)$ \\
\hline Pen-I & $\leq 0.03-8$ & 0.5 & 1 & 0.5 & $68.3(98.5)$ & $23.2(1.3)$ & $8.5(0.2)$ \\
\hline Pen-R & $0.5-16$ & 2 & 8 & 2 & $0.9(68.4)$ & $33.5(19.7)$ & $65.6(11.9)$ \\
\hline \multicolumn{8}{|l|}{ Cefuroxime } \\
\hline All & $\leq 0.25->16$ & $\leq 0.25$ & 8 & $\leq 0.25$ & 70.1 & 1.9 & 28.1 \\
\hline Pen-S & $\leq 0.25-4$ & $\leq 0.25$ & $\leq 0.25$ & $\leq 0.25$ & 99.8 & 0.1 & $0.0^{d}$ \\
\hline Pen-I & $\leq 0.25->16$ & 2 & 4 & 4 & 29.1 & 9.3 & 61.6 \\
\hline Pen-R & $2->16$ & 8 & 16 & 8 & 0 & 0 & 100 \\
\hline \multicolumn{8}{|l|}{ Ceftriaxone $^{e}$} \\
\hline All & $\leq 0.03-16$ & $\leq 0.03$ & 1 & $\leq 0.03$ & $82.7(96.4)$ & $13.7(2.1)$ & $3.6(1.5)$ \\
\hline Pen-S & $\leq 0.03-0.5$ & $\leq 0.03$ & 0.06 & $\leq 0.03$ & $100(100)$ & $0(0)$ & $0(0)$ \\
\hline Pen-I & $\leq 0.03-4$ & 0.5 & 1 & 0.5 & $89.7(99.3)$ & $9.6(0.6)$ & $0.7(0.1)$ \\
\hline Pen-R & $0.5-16$ & 1 & 2 & 1 & $8.1(78.6)$ & $73.5(12.4)$ & $21.4(9.0)$ \\
\hline \multicolumn{8}{|l|}{ Azithromycin } \\
\hline All & $\leq 0.06->32$ & $\leq 0.06$ & 6 & $\leq 0.06$ & 74.9 & 3.7 & 21.4 \\
\hline Pen-S & $\leq 0.06->32$ & $\leq 0.06$ & $\leq 0.12$ & $\leq 0.06$ & 94.5 & 0.7 & 4.8 \\
\hline Pen-I & $\leq 0.06->32$ & 1 & $>32$ & $\leq 0.06$ & 49.8 & 7.9 & 42.3 \\
\hline Pen-R & $\leq 0.06->32$ & 2 & $>32$ & $>32$ & 26.7 & 11.0 & 62.3 \\
\hline \multicolumn{8}{|l|}{ SXT } \\
\hline All & $\leq 0.03->4$ & 0.25 & $>4$ & 0.25 & 63.3 & 6.5 & 30.3 \\
\hline Pen-S & $\leq 0.03->4$ & 0.25 & 1 & 0.25 & 86.7 & 5.3 & 8.1 \\
\hline Pen-I & $0.06->4$ & 4 & $>4$ & $>4$ & 33.0 & 11.0 & 56.0 \\
\hline Pen-R & $0.06->4$ & 4 & $>4$ & $>4$ & 5.9 & 5.9 & 88.2 \\
\hline \multicolumn{8}{|l|}{ Levofloxacin } \\
\hline All & $\leq 0.008-32$ & 0.5 & 1 & 0.5 & 99.3 & 0.1 & 0.6 \\
\hline Pen-S & $\leq 0.008-16$ & 0.5 & 1 & 0.5 & 99.3 & 0.1 & 0.6 \\
\hline Pen-I & $0.25-16$ & 0.5 & 1 & 0.5 & 99.4 & 0 & 0.6 \\
\hline Pen-R & $0.25-32$ & 0.5 & 1 & 0.5 & 99.0 & 0.1 & 0.9 \\
\hline \multicolumn{8}{|l|}{ Moxifloxacin $^{f}$} \\
\hline All & $\leq 0.008-4$ & 0.12 & 0.12 & 0.12 & 99.5 & 0.4 & 0.1 \\
\hline Pen-S & $\leq 0.008-4$ & 0.12 & 0.12 & 0.12 & 99.5 & 0.5 & 0.1 \\
\hline Pen-I & $0.03-4$ & 0.12 & 0.12 & 0.12 & 99.7 & 0.2 & 0.1 \\
\hline Pen-R & $0.06-4$ & 0.12 & 0.12 & 0.12 & 99.2 & 0.5 & 0.3 \\
\hline \multicolumn{8}{|l|}{ Vancomycin } \\
\hline All & $\leq 0.12-1$ & 0.25 & 0.5 & 0.25 & 100 & $-^{h}$ & - \\
\hline Pen-S & $\leq 0.12-1$ & 0.25 & 0.5 & 0.25 & 100 & - & - \\
\hline Pen-I & $\leq 0.12-1$ & 0.25 & 0.5 & 0.25 & 100 & - & - \\
\hline Pen-R & $\leq 0.12-1$ & 0.25 & 0.5 & 0.25 & 100 & - & - \\
\hline
\end{tabular}

${ }^{a}$ A total of 3,189 isolates were penicillin susceptible (Pen-S), 952 were penicillin intermediate (Pen-I), and 799 were penicillin resistant (Pen-R).

${ }^{b} \mathrm{~S}$, susceptible; I, intermediate; R, resistant.

${ }^{c}$ NCCLS 1999 breakpoints (16) were used to interpret data for amoxicillin-clavulanate. Interpreted data utilizing NCCLS 2002 breakpoints (18) are shown in parentheses.

${ }^{d}$ Actual percent resistance was $0.02 \%$.

${ }^{e}$ NCCLS 1999 breakpoints (16) were used to interpret data for ceftriaxone. Interpreted data utilizing NCCLS 2002 interpretations (18) are shown in parentheses.

${ }^{f}$ NCCLS 2002 breakpoints (18) were used to interpret data for moxifloxacin.

${ }^{g} 50 \%$ and $90 \%$, MIC $_{50}$ and MIC $_{90}$, respectively.

$h_{-}$, no NCCLS criteria (18) available for I and R category intrepretations.

cin-resistant isolates appeared to increase significantly, no interpretive differences were noted for moxifloxacin, and modal MICs or MICs at which $90 \%$ of the isolates tested are inhibited $\left(\mathrm{MIC}_{90} \mathrm{~s}\right)$ for either fluoroquinolone remained constant (or decreased in the case of moxifloxacin) during both years (Table 2). Shifts are not likely to be clinically relevant for either fluoroquinolone. In addition, a recent study showed for $S$. pneumoniae that no strains for which levofloxacin MICs were 
TABLE 2. Longitudinal comparison of susceptibility of pneumococcal isolates from 1997-1998 $(n=3.352)$ and $1999(n=3.315)^{a}$

\begin{tabular}{|c|c|c|c|c|c|c|c|}
\hline \multirow{2}{*}{ Antimicrobial agent } & \multirow{2}{*}{ Yr } & \multicolumn{3}{|c|}{$\%$ of isolates $(\% \text { change })^{b}$} & \multirow{2}{*}{$P$ value ${ }^{c}$} & \multicolumn{2}{|c|}{ MIC $(\mu \mathrm{g} / \mathrm{ml})$} \\
\hline & & S (change) & I (change) & $\mathrm{R}$ (change) & & Mode & $90 \% f$ \\
\hline \multirow[t]{2}{*}{ Penicillin } & 1997-1998 & 65.4 & 21.9 & 12.7 & & $\leq 0.03$ & 2 \\
\hline & 1999 & $64.4(-1.0)$ & $19.2(-2.7)$ & $16.4(+3.7)$ & $<0.001$ & $\leq 0.06$ & 2 \\
\hline \multirow[t]{2}{*}{ Amoxicillin-clavulanate } & 1997-1998 & 83.0 & 8.6 & 8.4 & & $\leq 0.01$ & 1 \\
\hline & 1999 & $77.7(-5.3)$ & $10(+1.4)$ & $12.3(+3.9)$ & $<0.001$ & $\leq 0.03$ & 2 \\
\hline \multirow[t]{2}{*}{ Cefuroxime } & 1997-1998 & 73.6 & 3.4 & 23.0 & & $\leq 0.12$ & 4 \\
\hline & 1999 & $69.4(-4.2)$ & $1.9(-1.5)$ & $28.7(+5.7)$ & $<0.001$ & $\leq 0.25$ & 8 \\
\hline \multirow[t]{2}{*}{ Ceftriaxone } & 1997-1998 & 85.8 & 10.6 & 3.6 & & $\leq 0.01$ & 1 \\
\hline & 1999 & $82.4(-3.4)$ & $14.1(+3.5)$ & $3.5(-0.1)$ & 0.809 & $\leq 0.03$ & 1 \\
\hline \multirow[t]{2}{*}{ Azithromycin ${ }^{d}$} & 1997-1998 & 78.0 & 2.9 & 19.1 & & $\leq 0.03$ & 4 \\
\hline & 1999 & $74.6(-3.4)$ & $3.9(+1.0)$ & $21.5(+2.4)$ & 0.014 & $\leq 0.06$ & 8 \\
\hline \multirow[t]{2}{*}{ SXT } & 1997-1998 & 69.1 & 15.5 & 15.4 & & 0.12 & 4 \\
\hline & 1999 & $62.4(-6.7)$ & $6.8(-8.7)$ & $30.8(+15.4)$ & $<0.001$ & 0.25 & $>4$ \\
\hline \multirow[t]{2}{*}{ Levofloxacin } & 1997-1998 & 99.9 & 0 & 0.1 & & 0.5 & 1 \\
\hline & 1999 & $99.5(-0.4)$ & $0.1(+0.1)$ & $0.4(+0.3)$ & 0.017 & 0.5 & 1 \\
\hline \multirow[t]{2}{*}{ Moxifloxacin } & 1997-1998 & 99.9 & $<0.1$ & $<0.1$ & & 0.12 & 0.25 \\
\hline & 1999 & $99.6(-0.3)$ & $0.4(>+0.3)$ & $<0.1(-0.0)^{e}$ & 0.670 & 0.12 & 0.12 \\
\hline
\end{tabular}

${ }^{a}$ Only data from the 156 laboratories that participated in both studies and submitted a minimum of 10 isolates of pneumococci were included in the longitudinal analysis.

${ }^{b}$ Values illustrate the percent change in the susceptible (S), intermediate (I), or resistant (R), population between $1997-1998$ and 1999.

${ }^{c} P$ values were calculated based on the number of isolates resistant to each antimicrobial in each study period.

${ }^{d}$ Erythromycin was the macrolide tested in 1997-1998; azithromycin was tested in 1999.

${ }^{e}$ Actual percent change, $-0.03 \%$.

${ }^{f} 90 \%$, MIC $_{90}$.

$0.5 \mu \mathrm{g} / \mathrm{ml}$ and approximately only $5 \%$ of strains for which levofloxacin MICs were $1 \mu \mathrm{g} / \mathrm{ml}$ carried key mutations in QRDRs (4). As such, the MIC shifts measured (Fig. 1) are not likely to reflect mutational events in QRDRs and likely demonstrate the inherent weakness of statistical analysis in studies that incorporate a large number of isolates. In addition, the accepted artifact of \pm 1 dilution in MIC test results can also complicate comparative analysis of susceptibility test results. Comparison of MIC distributions from future years will confirm whether trends are real or artifactual.

Defining multiple drug resistance (MDR) as resistance to $\geq 3$ of the agents penicillin, ceftriaxone, erythromycin, SXT, vancomycin, and either levofloxacin or moxifloxacin and considering only isolates included in the longitudinal analysis, 212 of 3,352 isolates $(6.3 \%)$ from the 1997-1998 study, and 374 of 3,315 isolates $(11.3 \%)$ from 1999 exhibited MDR phenotypes, an increase of $5.0 \%(P<0.001)$. The number of organisms testing as susceptible to all compounds decreased from $57.2 \%$ $(n=1,919)$ to $53.8 \%(n=1,784)$ during the same period $(P<$ $0.005)$. Only four MDR isolates demonstrated resistance to levofloxacin, of which none were moxifloxacin resistant. Of the 35 isolates $(0.7 \%)$ nonsusceptible to levofloxacin (4 intermediate, 31 resistant), $26(0.5 \%)$ were nonsusceptible to moxifloxacin (21 intermediate, 5 resistant). Similar to the 11 isolates (0.2\%) from the 1997-1998 study for which levofloxacin MICs were $\geq 4 \mu \mathrm{g} / \mathrm{ml}$, single or combinations of classic QRDR alterations (7, 10, 11, 14, 20, 22, 23), namely GyrA (Ser81-Phe/Tyr or Glu85-Lys) with or without ParC alterations (Ser79-Phe/ Tyr, Asp83-Asn, Lys137-Asn) and with or without the ParE
Ile460-Val alteration, were detected in each fluoroquinoloneresistant isolate. No novel mutations were detected in the QRDRs in gyrA or parC in any isolates. The contribution of efflux was not measured, since all fluoroquinolone-resistant isolates possessed key QRDR mutations.

The 35 fluoroquinolone-nonsusceptible isolates varied considerably with respect to PFGE band profiles (difference of more than five bands) and were identical in only three instances. Two of these were isolates submitted by the same site. The third set consisted of two isolates submitted by hospitals in Illinois and New York. Aside from these isolates, few of the levofloxacin-resistant isolates demonstrated similarities of $>80 \%$ with the Dice coefficient to interpret isolate banding patterns (data not shown), and none could be considered closely related (24).

The $22.1 \%$ rate of nonsusceptibility recorded for penicillin reflects recent U.S. studies reported, including the TRUST $(24,25)$ and SENTRY $(5,19)$ studies. Similarly, the high levels of nonsusceptibility to macrolides $(25.1 \%)$ and SXT $(36.8 \%)$ reported confirm recent observations $(5,6,19,26)$. The two high-level $\beta$-lactam-resistant isolates represent unusual strains rarely isolated in the United States and suggest the need to test for higher-endpoint MICs. Longitudinal trending shows the continued emergence of penicillin-, amoxicillin-clavulanate-, cefuroxime-, macrolide-, and SXT-resistant S. pneumoniae. The increased incidence of MDR phenotypes demonstrates that several antimicrobial classes, including penicillin derivatives, cefuroxime, azithromycin, and SXT, are potentially able to select MDR isolates, an increasing problem that needs care- 

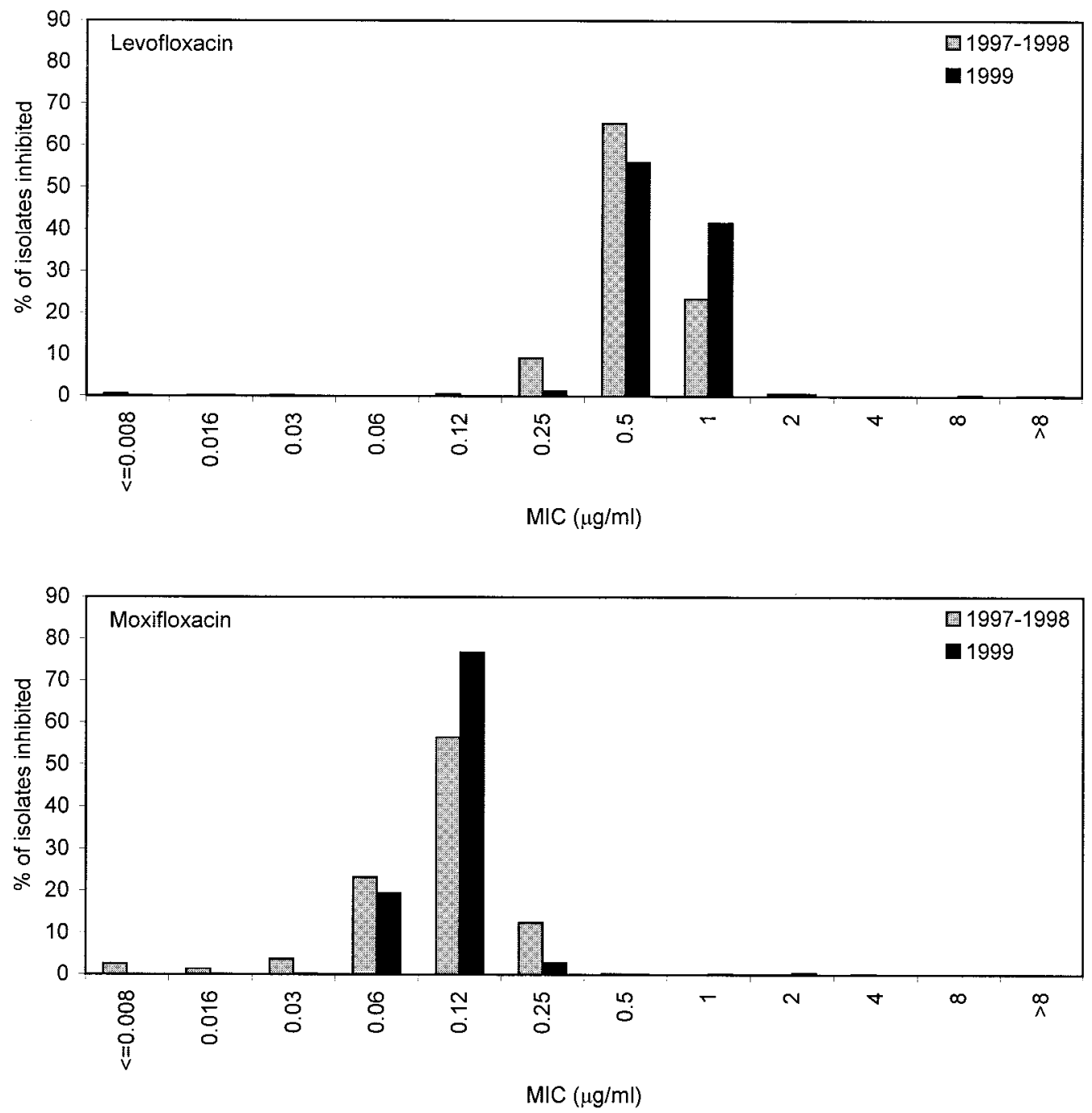

FIG. 1. Comparison of distributions of levofloxacin and moxifloxacin MICs from the 1997-1998 and 1999 studies. The achievable maximum concentrations of both drugs in serum following a single 400-mg (moxifloxacin) or 500-mg (levofloxacin) oral dose are $4.5 \pm 0.53$ and $5.1 \pm 0.8$ $\mu \mathrm{g} / \mathrm{ml}$, respectively (according to the package inserts).

ful study. In contrast, fluoroquinolone resistance was rarely associated with MDR phenotypes and occurred in only four MDR isolates, suggesting that, at least for now, fluoroquinolones may play a role in strategies to avoid the selection of MDR types. Apart from vancomycin, moxifloxacin and levofloxacin were the most active compounds in vitro. Analysis by PFGE of the 35 levofloxacin- or moxifloxacin-nonsusceptible isolates showed little evidence of clonal expansion of a fluoroquinolone-resistant strain of pneumococci, such as the socalled "type A" strain we identified as predominating during the 1997-1998 study (12).

We thank Bayer Corporation for providing funding for this study under the auspices of the LIBRA Surveillance program.

We express our appreciation to the many microbiologists and other laboratory personnel in each of the participating centers, without whose commitment these valuable studies would not be possible.

\section{REFERENCES}

1. Bartlett, J. G., and L. M. Mundy. 1995. Community-acquired pneumonia N. Engl. J. Med. 333:1618-1624.

2. Chen, D. K., A. McGeer, J. C. de Azavedo, and D. E. Low. 1999. Decreased susceptibility of Streptococcus pneumoniae to fluoroquinolones in Canada. Canadian Bacterial Surveillance Network. N. Engl. J. Med. 341:233-239.

3. Critchley, I. A., J. A. Karlowsky, D. C. Draghi, M. E. Jones, C. Thornsberry, K. Murfitt, and D. F. Sahm. 2002. Activities of faropenem, an oral $\beta$-lactam, against recent U.S. isolates of Streptococcus pneumoniae, Haemophilus influenzae, and Moraxella catarrhalis. Antimicrob. Agents Chemother. 46:550555.

4. Davies, T. A., A. Evangelista, S. Pfleger, K. Bush, D. F. Sahm, and R. Goldschmidt. 2002. Prevalence of single mutations in topoisomerase type II genes among levofloxacin-susceptible clinical strains of Streptococcus pneumoniae isolated in the United States in 1992 to 1996 and 1999 to 2000. Antimicrob. Agents Chemother. 46:119-124.

5. Doern, G. V., A. B. Brueggemann, H. Huynh, E. Wingert, and P. Rhomberg. 1999. Antimicrobial resistance with Streptococcus pneumoniae in the United States, 1997-98. Emerg. Infect. Dis. 5:757-765.

6. Doern, G. V., M. A. Pfaller, K. Kugler, J. Freeman, and R. N. Jones. 1998. Prevalence of antimicrobial resistance among respiratory tract isolates of Streptococcus pneumoniae in North America: 1997 results from the SENTRY antimicrobial surveillance program. Clin. Infect. Dis. 27:764-770. 
7. Fukuda, H., and K. Hiramatsu. 1999. Primary targets of fluoroquinolones in Streptococcus pneumoniae. Antimicrob. Agents Chemother. 43:410-412.

8. Goldsmith, C. E., J. E. Moore, P. G. Murphy, and J. E. Ambler. 1998 Increased incidence of ciprofloxacin resistance in penicillin-resistant pneumococci in Northern Ireland. J. Antimicrob. Chemother. 41:420-421.

9. Ho, P.-L., T.-L. Que, D. N.-C. Tsang, T.-K. Ng, K.-H. Chow, and W.-H. Seto. 1999. Emergence of fluoroquinolone resistance among multiply resistant strains of Streptococcus pneumoniae in Hong Kong. Antimicrob. Agents Chemother. 43:1310-1313.

10. Janoir, C., V. Zeller, M.-D. Kitzis, N. J. Moreau, and L. Gutmann. 1996. High-level fluoroquinolone resistance in Streptococcus pneumoniae requires mutations in parC and gyrA. Antimicrob. Agents Chemother. 40:2760-2764.

11. Jones, M. E., D. F. Sahm, N. Martin, S. Scheuring, P. Heisig, C. Thornsberry, K. Köhrer, and F.-J. Schmitz. 2000. Prevalence of gyrA, gyrB, parC and parE mutations in clinical isolates of Streptococcus pneumoniae with decreased susceptibilities to different fluoroquinolones and originating from worldwide surveillance studies during the 1997-1998 respiratory season. Antimicrob. Agents Chemother. 44:462-466.

12. Jones, M. E., A. M. Staples, I. Critchley, C. Thornsberry, P. Heinze, H. D. Engler, and D. F. Sahm. 2000. Benchmarking the in vitro activities of moxifloxacin and comparator agents against recent respiratory isolates from 377 medical centers throughout the United States. Antimicrob. Agents Chemother. 44:2645-2652.

13. McEllistrem, M. C., J. E. Stout, and L. H. Harrison. 2000. Simplified protocol for pulsed-field gel electrophoresis analysis of Streptococcus pneumoniae. J. Clin. Microbiol. 38:351-353.

14. Muñoz, R., and A. G. De la Campa. 1996. ParC subunit of DNA topoisomerase IV of Streptococcus pneumoniae is a primary target of fluoroquinolones and cooperates with DNA gyrase A subunit in forming resistance phenotype. Antimicrob. Agents Chemother. 40:2252-2257.

15. National Committee for Clinical Laboratory Standards. 2000. Methods for dilution antimicrobial susceptibility tests for bacteria that grow aerobically, 6th ed. Approved standard M7-A5. National Committee for Clinical Laboratory Standards, Wayne, $\mathrm{Pa}$.

16. National Committee for Clinical Laboratory Standards. 1999. Performance standards for antimicrobial susceptibility testing. Ninth informational supplement. M100-S9. National Committee for Clinical Laboratory Standards, Wayne, $\mathrm{Pa}$.

17. National Committee for Clinical Laboratory Standards. 2001. Performance standards for antimicrobial susceptibility testing. Eleventh informational supplement. M100-S11. National Committee for Clinical Laboratory Standards, Wayne, $\mathrm{Pa}$.

18. National Committee for Clinical Laboratory Standards. 2002. Performance standards for antimicrobial susceptibility testing. Twelfth informational supplement. M100-S12. National Committee for Clinical Laboratory Standards, Wayne, $\mathrm{Pa}$.

19. Odland, B. A., R. N. Jones, J. Verhoef, A. Fluit, and M. L. Beach. 1999. Antimicrobial activity of gatifloxacin (AM-1155, CG5501), and four other fluoroquinolones tested against 2,284 recent clinical strains of Streptococcus pneumoniae from Europe, Latin America, Canada, and the United States. The SENTRY Antimicrobial Surveillance Group (Americas and Europe). Diagn. Microbiol. Infect. Dis. 34:315-320.

20. Pan, X.-S., J. Ambler, S. Mehtar, and L. M. Fisher. 1996. Involvement of topoisomerase IV and DNA gyrase as ciprofloxacin targets in Streptococcus pneumoniae. Antimicrob. Agents Chemother. 40:2321-2326.

21. Sahm, D. F., D. E. Peterson, I. A. Critchley, and C. Thornsberry. 2000. Analysis of ciprofloxacin activity against Streptococcus pneumoniae after 10 years of use in the United States. Antimicrob. Agents Chemother. 44:25212524.

22. Taba, H., and N. Kusano. 1998. Sparfloxacin resistance in clinical isolates of Streptococcus pneumoniae: involvement of multiple mutations in gyrA and parC genes. Antimicrob. Agents Chemother. 42:2193-2196.

23. Tankovic, J., B. Perichon, J. Duval, and P. Courvalin. 1996. Contribution of mutations in gyrA and parC genes to fluoroquinolone resistance of mutants of Streptococcus pneumoniae obtained in vivo and in vitro. Antimicrob. Agents Chemother. 40:2505-2510.

24. Tenover, F. C., R. D. Arbeit, R. V. Goering, P. A. Mickelsen, B. E. Murray, D. H. Persing, and B. Swaminathan. 1995. Interpreting chromosomal DNA restriction patterns produced by pulsed-field gel electrophoresis: criteria for bacterial strain typing. J. Clin. Microbiol. 33:2233-2239.

25. Thornsberry, C., P. Ogilvie, J. Kahn, and Y. Mauriz. 1997. Surveillance of antimicrobial resistance in Streptococcus pneumoniae, Haemophilus influenzae, and Moraxella catarrhalis in the United States in 1996-1997 respiratory season. The Laboratory Investigator Group. Diagn. Microbiol. Infect. Dis. 29:249-257.

26. Thornsberry, C., M. E. Jones, M. Hickey, Y. Mauriz, J. Kahn, and D. F. Sahm. 1999. Resistance surveillance of Streptococcus pneumoniae, Haemophilus influenzae and Moraxella catarrhalis in the United States, 19971998. J. Antimicrob. Chemother. 44:749-759. 